\title{
Variáveis biométricas e componentes produtivos da cultura do trigo: mapeamento e correlação entre variáveis
}

Biometric variables and productive attributes of wheat: mapping and correlation among variables

\section{Osmar Henrique de Castro Pias', Júnior Melo Damian², Maurício Roberto Cherubin ${ }^{3}$ e Antônio Luis Santi $^{2}$}

Recebido em 07/06/2016 / Aceito em 04/08/2016

\section{RESUMO}

O objetivo deste estudo foi avaliar a relação existente entre variáveis biométricas quantificadas ao longo do desenvolvimento fenológico da cultura do trigo com o rendimento de grãos e componentes produtivos. O estudo foi realizado em uma área de 4,7 ha. Inicialmente a área foi georreferenciada e dividida em uma malha amostral de $50 \times 50 \mathrm{~m}$, resultando em 18 pontos amostrais. Em cada ponto amostral foram demarcadas três subparcelas de $2 \times 1 \mathrm{~m}$. Foi avaliado a emergência de plântulas aos 7 dias após a emergência (DAE) (estádio 1), número de plantas, número de afilhos, número de folhas por planta, comprimento radicular, altura, massa seca radicular, massa seca da parte aérea e número de nós aos 35 (estádio 5), 57 (estádio 8) e 80 (estádio 10,3) DAE. Os componentes produtivos número de espigas, peso de 1.000 grãos, número de grãos por espiga e produtividade foram quantificados aos 130 DAE (estádio 11,4). Diferenças biométricas em plantas de trigo a partir do estádio fenológico 8 afetaram os componentes produtivos da cultura. Plantas de trigo com elevada estatura (altura e número de nós) e reduzido comprimento radicular apresentaram maiores produtividades.

PALAVRAS-CHAVE: Triticum aestivum L., agricultura de precisão, componentes morfológicos, rendimento de grãos.

\footnotetext{
ABSTRACT

The aim of this study was to evaluate the relationship between biometric variables quantified along the phenological development of the wheat crop with

${ }^{1}$ Universidade Federal do Rio Grande do Sul, Porto Alegre, RS, Brasil.

${ }^{2}$ Universidade Federal de Santa Maria, Francisco Westphalen, RS, Brasil.

${ }^{3}$ Universidade de São Paulo, Piracicaba, SP, Brasil.

*Autor para correspondência <juniormelodamian@hotmail.com>
}

grain yield and production components. The study was carried out in an area of 4.7 ha. Initially the area was georeferenced and divided into a sampling grid of $50 \times 50 \mathrm{~m}$, resulting in 18 sample points. At each point three subplots of $2 \times 1 \mathrm{~m}$ were marked. The emergency of plants seven days after emergence (DAE) (stage 1), number of plants, number of tillers, number of leaves per plant, root length, height, root dry mass, dry mass of the aerial part and number of nodes at 35 (stage 5), 57 (stage 8) and 80 (stage 10.3) DAE were evaluated. The yield component was determined regarding the number of spikes; 1000 grain weight; number of grains per spike and grain yield at 130 DAE (stage 11.4). Biometric differences in wheat plants from phenological stage 8 affect the productive components of crop. Wheat plants higher (height and number of nodes) and reduced root length showed higher productivity.

KEYWORDS: Triticum aestivum L., precision agriculture, morphologic components, grain yield.

\section{INTRODUÇÃO}

O trigo (Triticum aestivum L.) destacase como a cultura de inverno mais produzida no Brasil. A área cultivada com a cultura na safra de 2015 foi de aproximadamente 2,5 milhões de ha com um rendimento de grãos médio de $2,3 \mathrm{Mg} \mathrm{ha}^{-1}$ (CONAB 2016). Embora, a cultura tenha reconhecida importância no agronegócio brasileiro o rendimento de grãos ainda é considerado baixo quando comparado a média mundial que chega a $3,0 \mathrm{Mg} \mathrm{ha}^{-1}$ (USDA 2014). O baixo rendimento de grãos obtido, aliado ao alto custo de produção tem resultado em uma 
baixa rentabilidade da cultura para grande parte dos produtores (BRUM \& MÜLLER 2008).

A eficiência produtiva da cultura do trigo é condicionada pela interação de um conjunto de fatores, entre os quais, se destacam a genética da semente, as condições edafoclimáticas, fertilidade do solo e o manejo cultural empregado (ZHAO et al. 2013, GAYLER et al. 2014). Nesse sentido, esforços de pesquisa têm sido feitos para elucidar a influência de atributos químicos (ZUCOLOTO et al. 2011) e físicos (PEREIRA et al. 2011) do solo no rendimento de grãos de trigo, porém, pouco se conhece sob as respostas biométricas da cultura ao longo de seu ciclo fenológico para a obtenção de elevados rendimentos (VESOHOSKI et al. 2011).

Nos países em que o rendimento de grãos do trigo é baixo, as condições climáticas têm sido consideradas como o principal fator limitante, sendo, portanto, fundamental avaliar as respostas fenológicas do trigo frente aos fatores do ambiente, na busca por técnicas de cultivo que melhorem o seu rendimento de grãos (KHAMSSI \& NAJAPHY 2012). O monitoramento espaço-temporal dos atributos fenológicos requer o uso de técnicas e tecnologias que possibilitem o fornecimento de informações em tempo hábil para possíveis intervenções, visando melhorias no manejo desses atributos para a cultura. Neste sentido, novas tecnologias têm se difundido, dentre as quais se destaca a agricultura de precisão (AP) (RODRIGUEZ-MORENO et al. 2014). Esta ferramenta tem como premissa básica a detecção e o estudo da variação existente de cada atributo constituinte do sistema solo/planta com o ambiente de produção, afim de que se possibilite o manejo localizado dos cultivos (GEMTOS et al. 2013, MAHAJAN et al. 2014).

A hipótese que fundamenta este trabalho é de que as variáveis biométricas da cultura do trigo, a partir de determinado estágio fenológico, apresentam alta relação com o rendimento de grãos. Neste sentido, o objetivo deste estudo foi avaliar a relação existente entre variáveis biométricas quantificadas ao longo do desenvolvimento fenológico da cultura do trigo com o rendimento de grãos e componentes produtivos.

\section{MATERIAL E MÉTODOS}

$\mathrm{O}$ estudo foi conduzido em uma área manejada sob o sistema de plantio direto há mais de 15 anos, localizada no município de Palmeira das Missões,
RS (Latitude 28 72'62" S e Longitude 69¹4'34" O), com altitude média de $600 \mathrm{~m}$. O clima da região segundo a classificação de Köeppen é do tipo Cfa, temperado chuvoso, com precipitação pluvial média anual elevada, oscilando entre 1.800 e 2.100 $\mathrm{mm}$, distribuída regularmente ao longo do ano e subtropical do ponto de vista térmico. A temperatura média anual é em torno $18{ }^{\circ} \mathrm{C}$, com máximas no verão podendo atingir $41^{\circ} \mathrm{C}$ e mínimas no inverno atingindo valores inferiores a $0{ }^{\circ} \mathrm{C}$ (MALUF 2000). Os dados de temperatura e precipitação pluvial durante a condução do experimento, obtidos pela estação meteorológica do INMET localizada em Palmeira das Missões a 15 $\mathrm{km}$ da área experimental são apresentados na Figura 1. O solo do local é classificado como Latossolo Vermelho distrófico, segundo as metodologias citadas por SANTOS et al. (2013). As características químicas e físicas do solo na camada de 0,00 - 0,10 $\mathrm{cm}$, no momento da instalação do experimento, foram: argila: $618 \mathrm{~g} \mathrm{~kg}^{-1}$, silte: $45 \mathrm{~g} \mathrm{~kg}^{-1}$, areia: 337 $\mathrm{g} \mathrm{kg}^{-1}$, matéria orgânica: $31,7 \mathrm{~g} \mathrm{~kg}^{-1}$, potássio: 125,0 $\mathrm{mg} \mathrm{dm}{ }^{-3}$, fósforo: $7,61 \mathrm{mg} \mathrm{dm}^{-3}, \mathrm{pH}$ em água: $5,2 \mathrm{e}$ saturação de bases: $54,5 \%$.

A área experimental de 4,7 ha foi inicialmente dividida em uma malha amostral de 50 × $50 \mathrm{~m}$, resultando em 18 pontos amostrais. A semeadura da cultura ocorreu no dia 14/06/2012, com a cultivar de trigo Quartzo que apresenta um ciclo médio de 128 dias, no espaçamento de $0,20 \mathrm{~m}$ entre linhas e na densidade de 330 sementes $\mathrm{m}^{-2}$. A adubação de base foi distribuída em taxa fixa utilizando-se o fertilizante de formulação 15-20-30 $\left(\mathrm{N}_{-} \mathrm{P}_{2} \mathrm{O}_{5}-\mathrm{K}_{2} \mathrm{O}\right)$ na dose de $250 \mathrm{~kg} \mathrm{ha}^{-1}$. A adubação de $\mathrm{N}$ em cobertura foi realizada com aplicação de ureia (45-00-00) na dose de $150 \mathrm{~kg} \mathrm{ha}^{-1}$ quando a cultura atingiu o estádio 1, apresentando de 3 a 5 folhas segundo escala de LARGE (1954). Os demais tratos culturais foram realizados seguindo-se as recomendações técnicas para a cultura (COMISSÃO 2011).

Anteriormente à emergência da cultura, foram demarcadas em um raio de $5 \mathrm{~m}$ de cada ponto amostral, três subparcelas de $2 \times 1 \mathrm{~m}$, sendo assim, utilizou-se a médias das três subparcelas para representar o valor de cada ponto amostral. A avaliação da emergência (EMER) da cultura foi realizada 15 dias após a semeadura (estádio 1), quantificando-se as plântulas emergidas em $1 \mathrm{~m}^{2}$ de cada subparcela. Aos 35 (estádio 5), 57 (estádio 8) e aos $80 \mathrm{DAE}$ (estádio 10,3), as plantas presentes em $1 \mathrm{~m}$ de cada subparcela foram avaliadas da seguinte maneira: inicialmente 


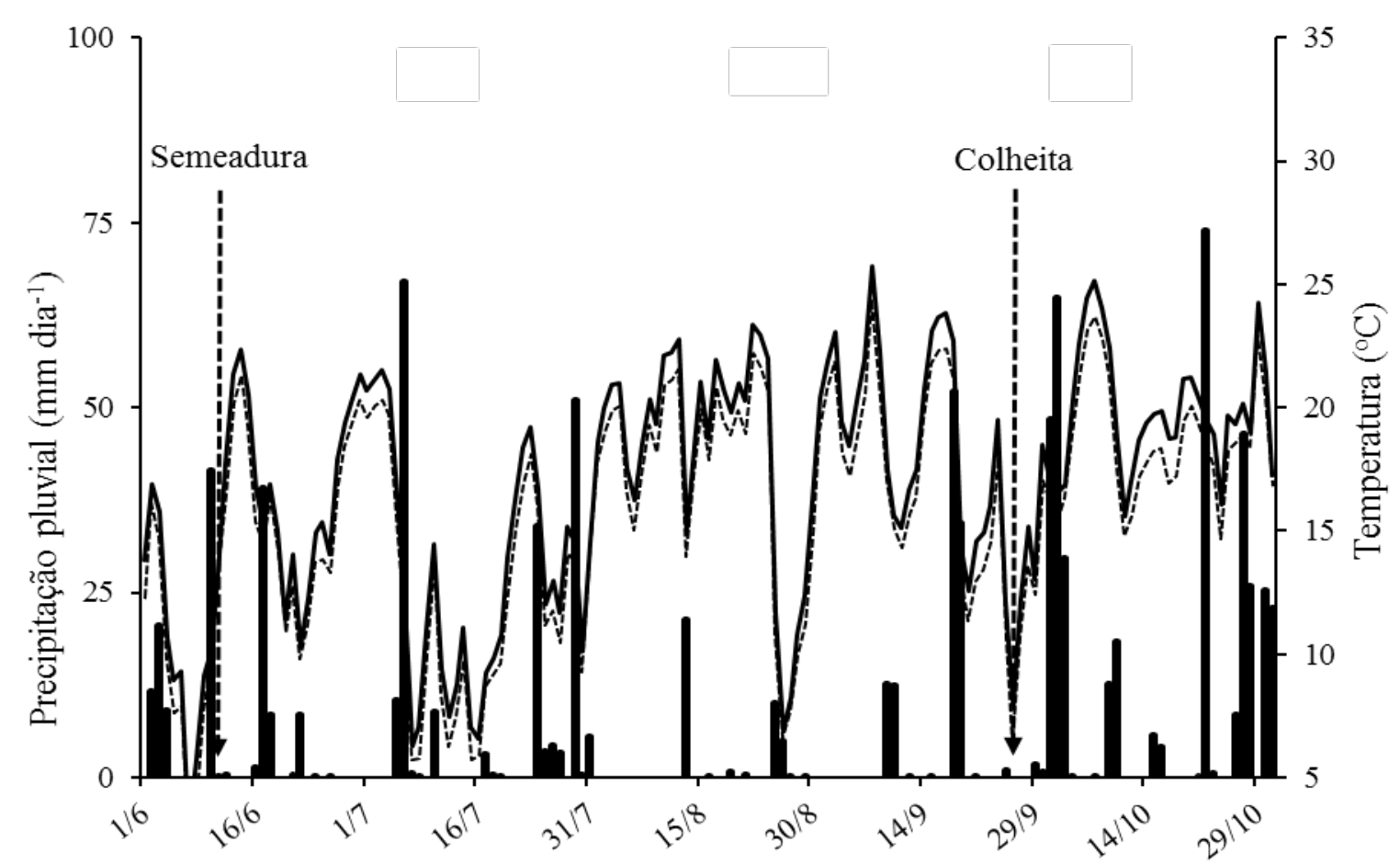

Figura 1 - Precipitação pluvial ( | ), temperaturas máxima (-) e temperatura mínima ( $\cdots)$, durante o período do experimento a campo em 2012. Palmeira das Missões, RS. 2016.

Figure 1 - Precipitation ( | ), maximum temperature (-) and minimum temperature (...), during the experiment period in the field in 2012. Palmeira das Missões, RS. 2016.

se fez a contagem do número de plantas (NP), na sequência procedeu-se a separação do sistema radicular e da parte aérea das plantas. O sistema radicular foi coletado manualmente por meio de uma pá de corte, sendo posteriormente, todo o sistema radicular lavado em água corrente com a ajuda de uma peneira. Após a lavagem escolheu-se ao acaso o sistema radicular e parte aérea de cinco plantas de cada subparcela (totalizando 15 plantas ponto ${ }^{-1}$ ) para mensuração do comprimento radicular (CR) e a altura (ALT) com régua graduada; número de afilhos (NA); número de nós $(\mathrm{NN})$ e número de folhas $(\mathrm{NF})$. Após as avaliações de contagem e medição, toda a parte aérea e radicular dos três metros coletados, foram acondicionadas em embalagens de papel e alocados em estufa de circulação de ar forçada a $65^{\circ} \mathrm{C}$ até a obtenção de peso constante. Em seguida, procedeu-se a pesagem das amostras em balança de precisão para a quantificação da massa seca radicular (MSR) $\left(\mathrm{kg} \mathrm{m}^{-1}\right)$ e da parte aérea (MSPA) $\left(\mathrm{kg} \mathrm{m}^{-1}\right)$ da cultura do trigo.

Quando a cultura atingiu a maturação (estádio 11,4) no dia 27/10/2012, realizou-se, de forma manual, a colheita de $1 \mathrm{~m}^{2}$ das subparcelas de cada ponto amostral. Na sequência, em laboratório, se quantificou o número de espigas $(\mathrm{NE})\left(\mathrm{m}^{-2}\right)$; peso médio de 1.000 grãos (P1000), obtido pela contagem de 100 grãos com três repetições; número médio de grãos espiga $^{-1}$ (NGE), obtido por meio da seguinte fórmula (peso de sementes em $1 \mathrm{~m}^{2} /$ peso de 1.000 grãos) / número de espigas $\mathrm{m}^{-2}$ ) e rendimento de grãos (REND) $\left(1 \mathrm{~m}^{2}\right)$, corrigindo-se os valores para uma umidade de $13 \%$ e extrapolando-os para $\mathrm{kg} \mathrm{ha}^{-1}$.

Os dados obtidos foram submetidos à análise estatística descritiva, calculando-se as medidas de posição (mínima, média e máxima) e de dispersão (coeficiente de variação, assimetria e curtose). Os valores de coeficiente de variação (CV) foram classificados como baixo $(\mathrm{CV} \leq 10 \%)$, médio $(10 \leq \mathrm{CV} \leq 20 \%)$, alto $(20 \leq \mathrm{CV} \geq 30 \%)$ e muito alto $(\mathrm{CV} \geq 30 \%)$ conforme descrito por GOMES \& GARCIA (2002). A hipótese de normalidade foi avaliada pelo teste $\mathrm{W}(\mathrm{p} \leq 0,05)$. Para avaliar a relação existente entre os parâmetros avaliados, submeteramse os dados à análise de matriz de correlação linear de Pearson $(p>0,05)$. Todas as análises foram realizadas com auxílio do software computacional Statistical Analysis System - SAS 8.0 (SAS INSTITUTE 1991). Posteriormente, para melhor visualização dos resultados, com auxílio do software CR-Campeiro 7 (Departamento de Geomática, Universidade Federal 
de Santa Maria, RS) os dados foram especializados em mapas temáticos. Devido ao reduzido número de pontos avaliados $(\mathrm{n}<50)$, impossibilitou-se o uso de análises geoestatísticas para aferições da variabilidade espacial das variáveis (WEBSTER \& OLIVER 2007), procedendo-se, portanto a interpolação dos dados pelo inverso do quadrado da distância que apresentam melhores resultados conjuntos de dados menores (COELHO et al. 2009).

\section{RESULTADOS E DISCUSSÃO}

Os resultados da análise estatística descritiva dos dados estão apresentados na Tabela 1. Observase que a média de emergência de plântulas foi de 273 plantas $\mathrm{m}^{-2}$, com um $\mathrm{CV}$ classificado como baixo (CV=6,5\%) (GOMES \& GARCIA 2002). Na primeira avaliação, aos $35 \mathrm{DAE}$ (estádio 5), o atributo que menos apresentou variação foi a altura de plantas com um $\mathrm{CV}$ de 5,6\%. A maior variação foi observada para a MSR $(\mathrm{CV}=21,9 \%)$ que foi classificada como alta. O sistema radicular das plantas apresenta uma elevada sensibilidade aos fatores ambientais, sendo que qualquer tipo e intensidade de estresse (hídrico, nutricional, etc.) induzirá a planta a desenvolver um maior sistema radicular, visando mitigar tal efeito. As demais variáveis biométricas apresentaram CVs médios $(10 \leq \mathrm{CV} \leq 20 \%)$. Nessa avaliação todas as variáveis seguiram distribuições normais de seus dados. A fase de elongação e emborrachamento da cultura do trigo apresentam elevada importância, devendo-se monitorar a variação dos atributos fenológicos, já que, durante esse período é definida parte importante do potencial produtivo da cultura do trigo, onde a elevação da variação pode comprometer o rendimento de grãos da cultura (LONGNECKER et al. 1993).

Na segunda avaliação aos 57 DAE (estádio 8) todas as variáveis com exceção da MSR e o NF que apresentaram CVs médios, apresentaram $\mathrm{CV}$ classificado como alto. A ALT de plantas, NN e NA não apresentaram distribuição normal, o que pode ser comprovado pelos elevados valores de assimetria e curtose (distantes de zero).

Aos 80 DAE (estádio 10,3), na terceira avaliação, todas as variáveis apresentam $\mathrm{CV}$ situados entre baixo e médio, sendo todos com ocorrência de distribuição normal dos dados, demonstrando-se uma menor variação dos atributos fenológicos do trigo.

Para os componentes do rendimento de grãos todas as variáveis apresentaram de baixa a média variação e distribuições normais. A maior variação dos dados foi observada para REND que apresentou um CV de 17,4\% sendo classificado como médio, corroborando com AMADO et al. (2007) que encontraram $\mathrm{CV}$ de $16 \%$ trabalhando com trigo em sistema de plantio direto. O REND médio foi de 2.611 $\mathrm{kg} \mathrm{ha}^{-1}$, contudo observam-se pontos com valores discrepantes aonde se obteve REND superior a 3.400 $\mathrm{kg} \mathrm{ha}^{-1}$ e abaixo de $2.000 \mathrm{~kg} \mathrm{ha}^{-1}$, demonstrando assim a existência de variação do REND mesmo em áreas pequenas.

Dentre os componentes do rendimento, o atributo que melhor se correlacionou com as variáveis biométricas do trigo aos 35 DAE foi o P1000, com correlação positiva com a ALT $(r=0,77)$ e MSPA das plantas $(\mathrm{r}=0,56)$ (Tabela 2). Apesar de o P1000 ser definido após a antese, a relação positiva entre as variáveis biométricas aos $35 \mathrm{DAE}$ e o atributo $\mathrm{P} 1000$, podem indicar que as plantas com maior desenvolvimento na fase inicial desenvolveram características benéficas com efeitos indiretos na cultura durante o seu ciclo reprodutivo. Esse resultado pode também estar relacionado com a suscetibilidade do P1000 grãos aos fatores ambientais, por ser uma variável de baixa herdabilidade genética (VESOHOSKI et al. 2011), e dessa forma, ocorrer correlações significativas ao acaso com alguns atributos, sem necessariamente haver relação de causa e efeito.

A EMER de plântulas apresentou correlação positiva com o NPL $(r=0,57)$ e MSPA $(r=0,64)$. O NPL no momento da avaliação (35 DAE) apresentou correlação negativa com o NA $(\mathrm{r}=-0,56)$ e NF $(\mathrm{r}=-0,61)$ o que já era um resultado esperado. Consequentemente com a redução do NPL houve aumento do NA e do NF sendo que essas últimas variáveis apresentaram elevada relação $(\mathrm{r}=0,92)$.

A partir dos mapas temáticos interpolados da emergência e das variáveis biométricas do trigo aos 35 DAE (Figura 2), pode-se observar que nas zonas onde a EMER e o NPL foi elevado (centro-sudoeste do mapa), houve redução do NA e do NF, reforçando os resultados obtidos na análise de correlação. Esse resultado comprova a elevada plasticidade da cultura do trigo onde é capaz de compensar possível desuniformidade da população de plantas pelo aumento do NA e consequentemente NF. 
Tabela 1 - Resumo da análise estatística descritiva dos dados de emergência, variáveis biométricas aos 35, 57 e 80 dias após a emergência e componentes de rendimento da cultura do trigo. Palmeira das Missões, RS. 2016.

Table 1 - Summary of statistical analysis of emergency data, biometric variables at 35, 57 and 80 days after emergence and profit components of the wheat crop. Palmeira das Missões, RS. 2016.

\begin{tabular}{|c|c|c|c|c|c|c|c|}
\hline \multirow{2}{*}{ Variável } & \multirow{2}{*}{ Mínimo } & \multirow{2}{*}{ Média } & \multirow{2}{*}{ Máximo } & \multicolumn{3}{|c|}{ Coeficiente } & \multirow{2}{*}{ Teste $(\mathrm{W})^{(1)}$} \\
\hline & & & & Variação & Simetria & Curtose & \\
\hline EMER & 240,00 & 272,73 & 310,00 & 6,50 & 0,48 & 0,61 & $0,95^{\mathrm{ns}}$ \\
\hline \multicolumn{8}{|c|}{ Variável biométrica $1^{\mathrm{a}}$ avaliação (35 $\mathrm{DAE}$ - estádio 1 ) } \\
\hline NPL & 230,00 & 295,56 & 390,00 & 15,80 & 0,64 & $-0,23$ & $0,94^{\mathrm{ns}}$ \\
\hline NA & 1,53 & 2,35 & 3,20 & 17,67 & $-0,02$ & 0,22 & $0,99^{\mathrm{ns}}$ \\
\hline NF & 5,93 & 7,67 & 10,07 & 12,90 & 0,38 & 0,99 & $0,96^{\mathrm{ns}}$ \\
\hline $\mathrm{CR}$ & 7,71 & 10,52 & 12,09 & 11,12 & $-0,73$ & 0,32 & $0,95^{\mathrm{ns}}$ \\
\hline ALT & 21,95 & 25,20 & 27,78 & 5,61 & $-0,24$ & 0,47 & $0,98^{\text {ns }}$ \\
\hline MSR & 121,67 & 173,94 & 240,00 & 21,91 & 0,45 & $-1,16$ & $0,92^{\text {ns }}$ \\
\hline MSPA & 362,33 & 540,57 & 738,67 & 17,14 & $-0,07$ & 0,53 & $0,93^{\text {ns }}$ \\
\hline \multicolumn{8}{|c|}{ Variável biométrica $2^{\mathrm{a}}$ avaliação (57 DAE - estádio 8) } \\
\hline NPL & 213,33 & 247,63 & 316,67 & 10,21 & $-0,75$ & 0,04 & $0,93^{\text {ns }}$ \\
\hline NA & 2,47 & 2,97 & 4,20 & 13,82 & 1,45 & 3,61 & $0,87 *$ \\
\hline NF & 7,33 & 10,32 & 16,27 & 20,99 & 1,28 & 2,27 & $0,90^{\mathrm{ns}}$ \\
\hline $\mathrm{NN}$ & 1,80 & 2,23 & 3,27 & 15,46 & 1,48 & 2,90 & $0,87 *$ \\
\hline $\mathrm{CR}$ & 9,23 & 12,28 & 14,97 & 12,39 & $-0,03$ & $-0,04$ & $0,96^{\mathrm{ns}}$ \\
\hline ALT & 41,63 & 51,05 & 76,72 & 14,78 & 2,37 & 8,00 & $0,78 *$ \\
\hline MSR & 278,00 & 414,91 & 655,00 & 20,02 & 1,33 & 3,24 & $0,91^{\mathrm{ns}}$ \\
\hline MSPA & 1.410 & 2.125 & 3.012 & 18,14 & 0,22 & 0,81 & $0,97^{\mathrm{ns}}$ \\
\hline \multicolumn{8}{|c|}{ Variável biométrica $3^{\mathrm{a}}$ avaliação (80 DAE - estádio 10,3$)$} \\
\hline NPL & 193,33 & 275,00 & 353,33 & 15,68 & 0,00 & $-0,6$ & $0,99^{\text {ns }}$ \\
\hline NA & 1,07 & 1,58 & 2,00 & 14,50 & $-0,34$ & 0,20 & $0,98^{\text {ns }}$ \\
\hline NF & 2,73 & 4,23 & 5,07 & 17,10 & $-0,59$ & $-0,48$ & $0,92^{\text {ns }}$ \\
\hline $\mathrm{NN}$ & 3,73 & 4,26 & 4,60 & 5,65 & $-0,59$ & $-0,42$ & $0,93^{\mathrm{ns}}$ \\
\hline $\mathrm{CR}$ & 9,10 & 11,56 & 13,93 & 12,12 & $-0,15$ & $-0,92$ & $0,96^{\mathrm{ns}}$ \\
\hline ALT & 56,00 & 64,68 & 73,43 & 8,34 & 0,25 & $-1,09$ & $0,95^{\mathrm{ns}}$ \\
\hline MSR & 338,00 & 471,83 & 608,00 & 13,66 & $-0,13$ & 0,47 & $0,99^{\text {ns }}$ \\
\hline MSPA & 3.834 & 4.850 & 6.580 & 15,82 & 0,30 & $-0,19$ & $0,93^{\text {ns }}$ \\
\hline \multicolumn{8}{|c|}{ Componente do rendimento (130 DAE - estádio 11,4$)$} \\
\hline $\mathrm{NE}$ & 259,67 & 331,89 & 378,00 & 10,45 & 0,45 & $-0,62$ & $0,95^{\mathrm{ns}}$ \\
\hline P1000 & 23,56 & 25,92 & 27,90 & 4,16 & $-0,47$ & 0,11 & $0,97^{\mathrm{ns}}$ \\
\hline NGE & 24,93 & 30,22 & 34,63 & 8,79 & $-0,27$ & $-0,61$ & $0,98^{\mathrm{ns}}$ \\
\hline REND & 1.876 & 2.612 & 3.438 & 17,39 & $-0,09$ & $-0,63$ & $0,97^{\mathrm{ns}}$ \\
\hline
\end{tabular}

${ }^{(1)} \mathrm{W}=$ Teste de Shapiro-Wilk para distribuição normal, onde: $\left({ }^{*}\right)$ significativo em níveis de $\mathrm{p} \leq 0,05$, ${ }^{\text {(ns) }}$ não significativo. Quando for significativo indica que a hipótese para distribuição normal é rejeitada. EMER $=$ Emergência; NPL= Número de plantas; $\mathrm{NA}=$ Número de afilhos; $\mathrm{NF}=$ Número de folhas por planta; $\mathrm{CR}=$ Comprimento radicular; $\mathrm{ALT}=\mathrm{Altura}$; $\mathrm{MSR}=$ Massa seca radicular; $\mathrm{MSPA}=$ Massa seca de parte aérea; $\mathrm{NN}=$ Número de nós; $\mathrm{NE}=$ Número de espigas por $\mathrm{m}^{-2} ; \mathrm{P} 1000=$ Peso de 1.000 grãos; $\mathrm{NGE}=$ Número de grãos por espiga; $\mathrm{REND}=$ Rendimento de grãos . 
Tabela 2 - Correlação entre a emergência de plântulas, variáveis biométricas aos 35, 57 e 80 dias após a emergência e componentes de rendimento da cultura do trigo. Palmeira das Missões, RS. 2016.

Table 2 - Correlation between seedling emergence, biometric variables at 35, 57 and 80 days after emergence and yield components of crop wheat crop. Palmeira das Missões, RS. 2016.

\begin{tabular}{|c|c|c|c|c|c|c|c|c|c|c|c|}
\hline Variável & ALT & NA & $\mathrm{NF}$ & $\mathrm{CR}$ & MSR & MSPA & $\mathrm{NE}$ & P1000 & REND & NGE & EMER \\
\hline \multicolumn{12}{|c|}{ 1ª Avaliação (35 DAE - estádio 1) } \\
\hline NPL & $-0,32$ & $-0,56^{*}$ & $-0,61 *$ & 0,12 & 0,09 & 0,34 & $-0,35$ & $-0,16$ & $-0,28$ & $-0,16$ & $0,57 *$ \\
\hline ALT & - & 0,32 & 0,35 & 0,31 & 0,36 & $0,57 *$ & 0,23 & $0,77 *$ & 0,39 & 0,21 & 0,11 \\
\hline NA & - & - & $0,92 *$ & 0,30 & 0,42 & 0,25 & 0,11 & 0,26 & 0,13 & 0,14 & $-0,06$ \\
\hline NF & - & - & - & 0,27 & 0,38 & 0,26 & $-0,05$ & 0,31 & 0,12 & 0,28 & $-0,05$ \\
\hline CR & - & - & - & - & 0,17 & $0,62 *$ & 0,06 & 0,20 & 0,18 & 0,13 & 0,16 \\
\hline MSR & - & - & - & - & - & 0,41 & $-0,07$ & 0,46 & 0,08 & 0,15 & 0,30 \\
\hline MSPA & - & - & - & - & - & - & $-0,14$ & $0,56^{*}$ & 0,16 & 0,23 & $0,64 *$ \\
\hline \multicolumn{12}{|c|}{$2^{\mathrm{a}}$ Avaliação (57 DAE - estádio 8) } \\
\hline $\mathrm{NN}$ & 0,06 & 0,09 & 0,14 & $-0,42$ & $-0,08$ & 0,43 & 0,28 & 0,28 & 0,30 & 0,10 & 0,02 \\
\hline NPL & 0,13 & $-0,53 *$ & $-0,45$ & 0,02 & $-0,01$ & $-0,01$ & 0,18 & 0,06 & 0,22 & 0,14 & 0,24 \\
\hline ALT & - & $-0,25$ & 0,09 & $-0,42$ & 0,38 & 0,34 & 0,37 & 0,34 & 0,24 & $-0,06$ & 0,16 \\
\hline NA & - & - & 0,37 & $-0,19$ & $-0,08$ & 0,14 & $-0,01$ & $-0,40$ & $-0,11$ & $-0,05$ & $-0,16$ \\
\hline NF & - & - & - & $-0,36$ & 0,21 & 0,40 & 0,26 & 0,33 & 0,34 & 0,22 & $-0,18$ \\
\hline CR & - & - & - & - & $-0,32$ & $0,78^{*}$ & $-0,50^{*}$ & $-0,55^{*}$ & $-0,51 *$ & $-0,21$ & $-0,42$ \\
\hline MSR & - & - & - & - & - & $0,58 *$ & $-0,07$ & 0,34 & 0,12 & 0,21 & 0,09 \\
\hline MSPA & - & - & - & - & - & - & 0,32 & $0,49 *$ & 0,45 & 0,32 & 0,29 \\
\hline \multicolumn{12}{|c|}{$3^{\mathrm{a}}$ Avaliação (80 DAE - estádio 10,3 ) } \\
\hline NN & 0,45 & 0,11 & $-0,07$ & $-0,04$ & $0,52 *$ & $0,57^{*}$ & $0,42 *$ & 0,30 & $0,54^{*}$ & $0,49 *$ & 0,09 \\
\hline NPL & $-0,09$ & $-0,30$ & 0,26 & 0,28 & $-0,01$ & $0,52 *$ & $-0,01$ & $-0,17$ & $-0,03$ & 0,17 & $-0,21$ \\
\hline ALT & - & $-0,11$ & $-0,17$ & $-0,24$ & 0,06 & 0,37 & 0,41 & $0,73^{*}$ & $0,58 *$ & 0,41 & 0,25 \\
\hline NA & - & - & $0,76^{*}$ & 0,43 & 0,38 & 0,09 & $-0,35$ & $-0,21$ & $-0,32$ & $-0,19$ & $-0,01$ \\
\hline NF & - & - & - & $0,49 *$ & 0,11 & 0,12 & $-0,50^{*}$ & $-0,19$ & $-0,47^{*}$ & $-0,30$ & $-0,11$ \\
\hline CR & - & - & - & - & 0,26 & 0,34 & $-0,35$ & $-0,40$ & $-0,45^{*}$ & $-0,33$ & $-0,42$ \\
\hline MSR & - & - & - & - & - & $0,49 *$ & 0,09 & 0,11 & 0,18 & 0,29 & $-0,06$ \\
\hline MSPA & - & - & - & - & - & - & $-0,16$ & $-0,22$ & $-0,09$ & 0,11 & 0,04 \\
\hline \multicolumn{12}{|c|}{ Componentes do rendimento (130 DAE - estádio 11,4$)$} \\
\hline $\mathrm{NE}$ & - & - & - & - & - & - & - & 0,29 & $0,79 *$ & 0,29 & 0,03 \\
\hline P1000 & - & - & - & - & - & - & - & - & $0,61 *$ & 0,46 & 0,31 \\
\hline NGE & - & - & - & - & - & - & - & - & - & - & 0,23 \\
\hline REND & - & - & - & - & - & - & - & - & - & $0,77 *$ & 0,44 \\
\hline
\end{tabular}

*Correlação linear de Pearson significativa $(\mathrm{p}<0,10) . \mathrm{EMER}=$ Emergência; $\mathrm{NPL}=$ Número de plantas; $\mathrm{NA}=$ Número de afilhos; $\mathrm{NF}=$ Número de folhas por planta; $\mathrm{CR}=$ Comprimento radicular; $\mathrm{ALT}=$ Altura; $\mathrm{MSR}=$ Massa seca radicular; MSPA= Massa seca de parte aérea; $\mathrm{NN}=$ Número de nós; $\mathrm{NE}=$ Número de espigas; P1000= Peso de 1.000 grãos; NGE= Número de grãos por espiga; REND= Rendimento de grãos. 


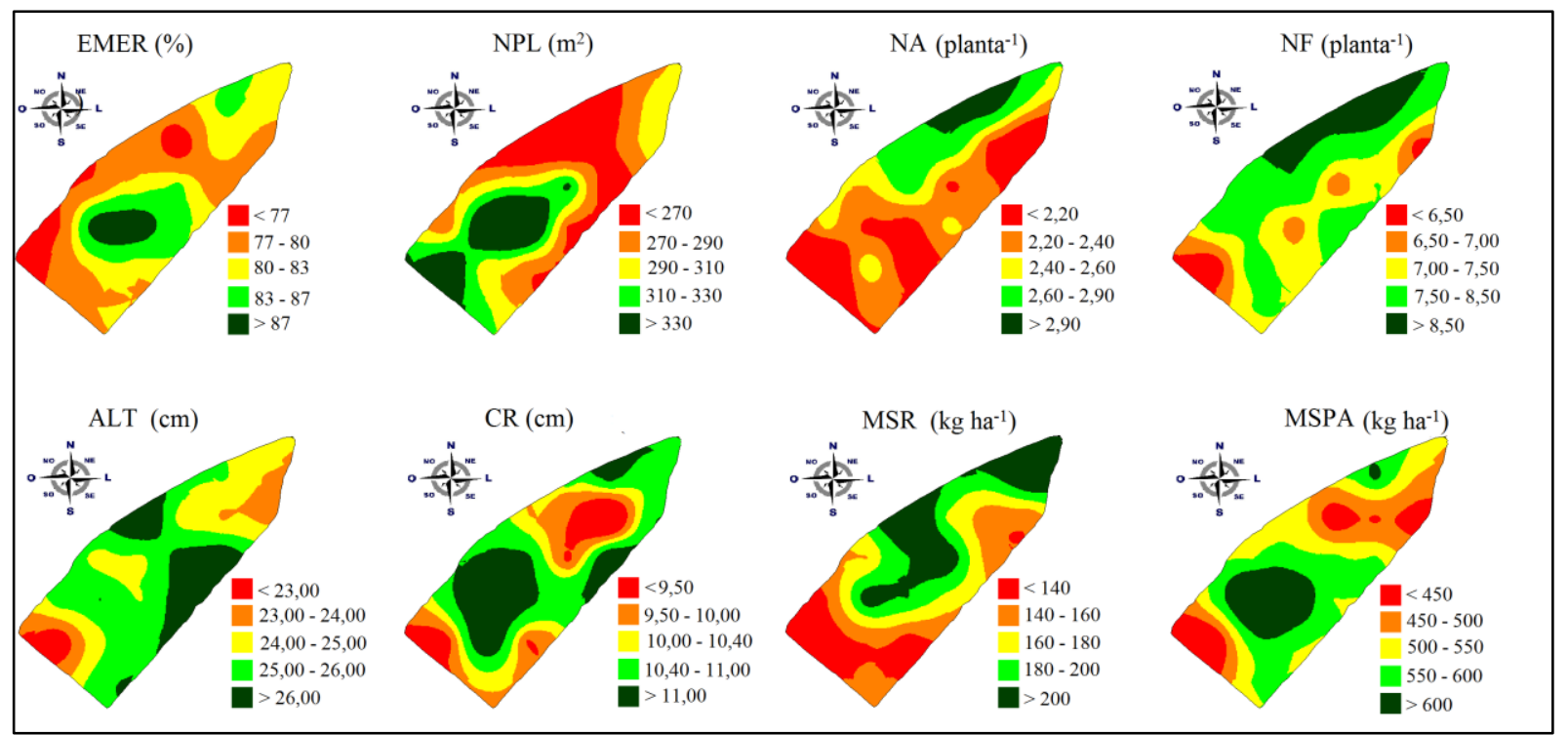

Figura 2 - Mapas temáticos da emergência (EMER), número de plantas (NPL), número de afilhos (NA), número de folhas por planta (NF), altura (ALT), comprimento de raízes (CR), massa seca de raízes (MSR) e massa seca de parte aérea (MSPA) da cultura do trigo aos 35 dias após a emergência. Palmeira das Missões, RS. 2016.

Figure 2 - Thematic maps of the number of nodes (NN), number of plants (NPL), number of tillers (NA), number of leaves per plant (NF), height (ALT), root length (CR), root dry weight (MSR) and dry mass of aerial part (MSPA) of wheat crop at 35 days after emergence. Palmeira das Missões, RS. 2016.

Aos 57 DAE (estádio 5) o P1000 apresentou correlação negativa com o $\mathrm{CR}(\mathrm{r}=-0,55)$ e positiva com a MSPA $(\mathrm{r}=0,49)$. A MSPA teve correlação positiva com a MSR $(\mathrm{r}=0,58)$ e $\mathrm{CR}(\mathrm{r}=0,78)$ (Tabela 2). O REND e o NE tiveram correlações negativas com o CR apresentando valores de $-0,51$ e -0,50, respectivamente. O NPL também apresentou correlação negativa com o NA $(r=-0,53)$. Plantas que estão passando por algum tipo de estresse apresentam como resposta um maior desenvolvimento do sistema radicular visando explorar uma maior área no solo para a busca por nutrientes e água. Um estresse que ocorreu na área experimental e que pode justificar os presentes resultados é o nutricional, devido ao baixo $\mathrm{pH}$ em alguns pontos da lavoura, que pode ter reduzido a disponibilidade de alguns nutrientes, houve correlação de $-0,52(\mathrm{p} \leq 0,05)$ e $-0,78(\mathrm{p} \leq 0,01)$ entre os valores de $\mathrm{pH}$ e o comprimento radicular na segunda e terceira avaliação, respectivamente (danos não apresentados). Em trabalho realizado por DAMIAN et al. (2016) nessa mesma área para essa mesma safra estudou-se a influência dos atributos químicos e físicos do solo sob a produtividade da cultura do trigo onde contatou-se que o $\mathrm{pH}$ teve uma variação na área amostral de 4,6 a 5,6, sendo seus valores altamente correlacionados a produtividade de grãos $(\mathrm{R} 2=0,76, \mathrm{p} \leq 0,01 \%)$. $\mathrm{O} \mathrm{pH}$ ideal para a cultura do trigo é de 5,5 - 6,0 (CQFS-RS/SC 2004). Nesse sentido, RODRIGUEZ-MORENO et al. (2014) citam que a implementação de interpretações de respostas fenológicas espaciais das plantas, nas tomadas de decisões sobre os manejos a serem adotados nas culturas, pode elevar o rendimento de grãos e reduzir danos ao meio ambiente.

BREDEMEIER et al. (2001), estudando o efeito da qualidade da semente de trigo no desenvolvimento e rendimento de grãos da cultura, observaram elevada influência do número de afilhos no rendimento de grãos. Os autores descrevem que caso os afilhos não apresentem desenvolvimento adequado, repercutirá em efeitos diretos nos demais atributos fenológicos da cultura, prejudicando o potencial produtivo da planta. A EMER não teve correlação significativa com o NPL na avaliação aos 57 DAE. Na Figura 3, mapas temáticos interpolados mostram a relação existente entre os atributos MSPA e MSR com o rendimento de grãos do trigo.

$\mathrm{Na} 3^{\mathrm{a}}$ avaliação das variáveis biométricas da cultura do trigo (80 DAE) ocorreram as maiores correlações com o REND, sendo que houve relação positiva com o $\mathrm{NN}(\mathrm{r}=0,54)$ e ALT $(\mathrm{r}=0,58)$ de plantas e negativas com o $\mathrm{CR}(\mathrm{r}=-0,45)$ e o NF $(\mathrm{r}=-0,47)$. A 


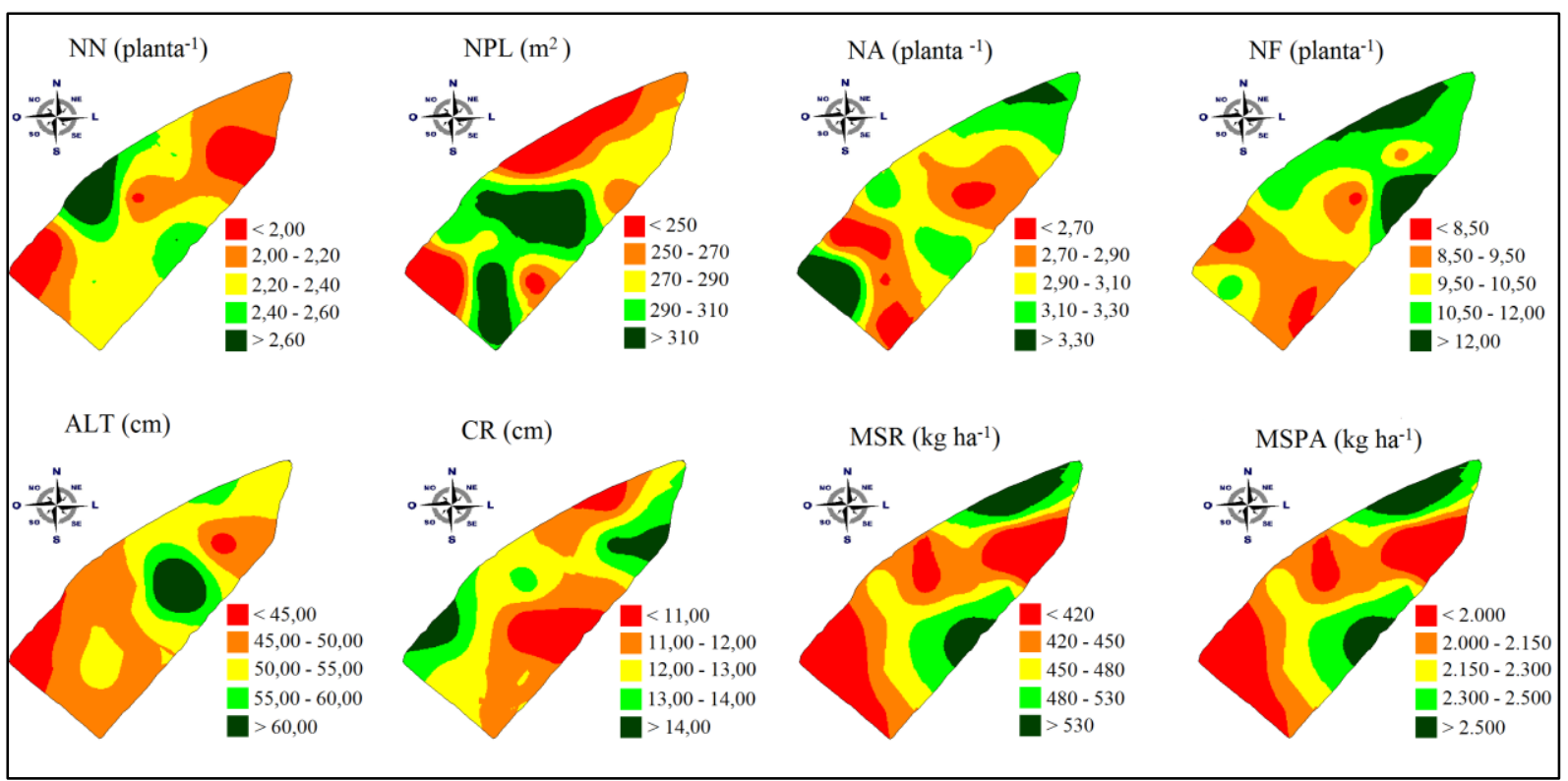

Figura 3 - Mapas temáticos do número de nós (NN), número de plantas (NPL), número de afilhos (NA), número de folhas por planta (NF), altura (ALT), comprimento radicular (CR), massa seca radicular (MSR) e massa seca de parte aérea (MSPA) da cultura do trigo aos 57 dias após a emergência. Palmeira das Missões, RS. 2016.

Figure 3 - Thematic maps of the number of nodes (NN), number of plants (NPL), number of tillers (NA), number of leaves per plant (NF), height (ALT), root length (CR), root dry weight (MSR) and dry mass of aerial part (MSPA) of wheat crop at 57 days after emergence. Palmeira das Missões, RS. 2016.

ALT de plantas ainda apresentou elevada correlação com o P1000 grãos $(\mathrm{r}=0,73)$. O NN correlacionouse com o $\mathrm{NGE}(\mathrm{r}=0,43)$. O NE apresentou relação inversa com o NF $(r=-0,50)$.

A MSPA apresentou correlação positiva com a MSR $(r=0,49), N N(r=0,57)$ e NPL $(r=0,52)$. A produção de MSR apresentou correlação positiva com o $\mathrm{NN}(\mathrm{r}=0,52)$. O NF apresentou correlação positiva com o $\mathrm{CR}(\mathrm{r}=0,49)$. Novamente o NA foi correlacionado com o NF $(r=0,76)$. Na Figura 4, são apresentados os mapas temáticos das variáveis biométricas da cultura do trigo aos $80 \mathrm{DAE}$, podendose visualizar a distribuição da variação dos atributos avaliados.

Houve correlação positiva entre REND e todos os componentes do rendimento. A maior correlação foi obtida entre o NE e REND com valor de $r$ de 0,79 . Entre REND e o NGE também foi verificada forte correlação positiva $(\mathrm{r}=0,77)$. Esses resultados são semelhantes aos obtidos por VIEIRA et al. (2007) e GONDIM et al. (2008).

Entre o P1000 e REND houve correlação positiva com um $r$ de 0,61 . Na Figura 5 são apresentados os mapas temáticos dos componentes de rendimento da cultura do trigo. Pode-se observar que para os dados de REND houve diferença média entre as classes de $600 \mathrm{~kg} \mathrm{ha}^{-1}$. De acordo com o mapa temático, o atributo que teve menor variação foi o P1000 grãos. Em um trabalho semelhante a este para a cultura da soja, DALCHIAVON \& CARVALHO (2012) observaram as maiores correlações com o rendimento de grãos para as variáveis número de vagens por planta e a massa de grãos por planta. Esses mesmos autores ainda citam que após avaliado qual a variável com maior contribuição para o rendimento de grãos (neste estudo o NE), é possível quantificar o rendimento de grãos de maneira indireta a campo com um elevado grau de confiabilidade.

Apesar de o NE ter sido a variável mais bem correlacionada com o rendimento de grãos de trigo, essa variável é determinada apenas quando a cultura já está em um estágio fenológico avançado. Sendo assim, as variáveis de estatura de plantas (altura e NN) que se correlacionaram com o REND apresentam maior importância, pois são determinadas em estágios iniciais, podendo-se utilizar ferramentas que detectem os locais de menor desenvolvimento propicie a aplicações de fertilizantes (N) para melhorar o desenvolvimento da cultura, visando uma homogeneização da produtividade no talhão. 


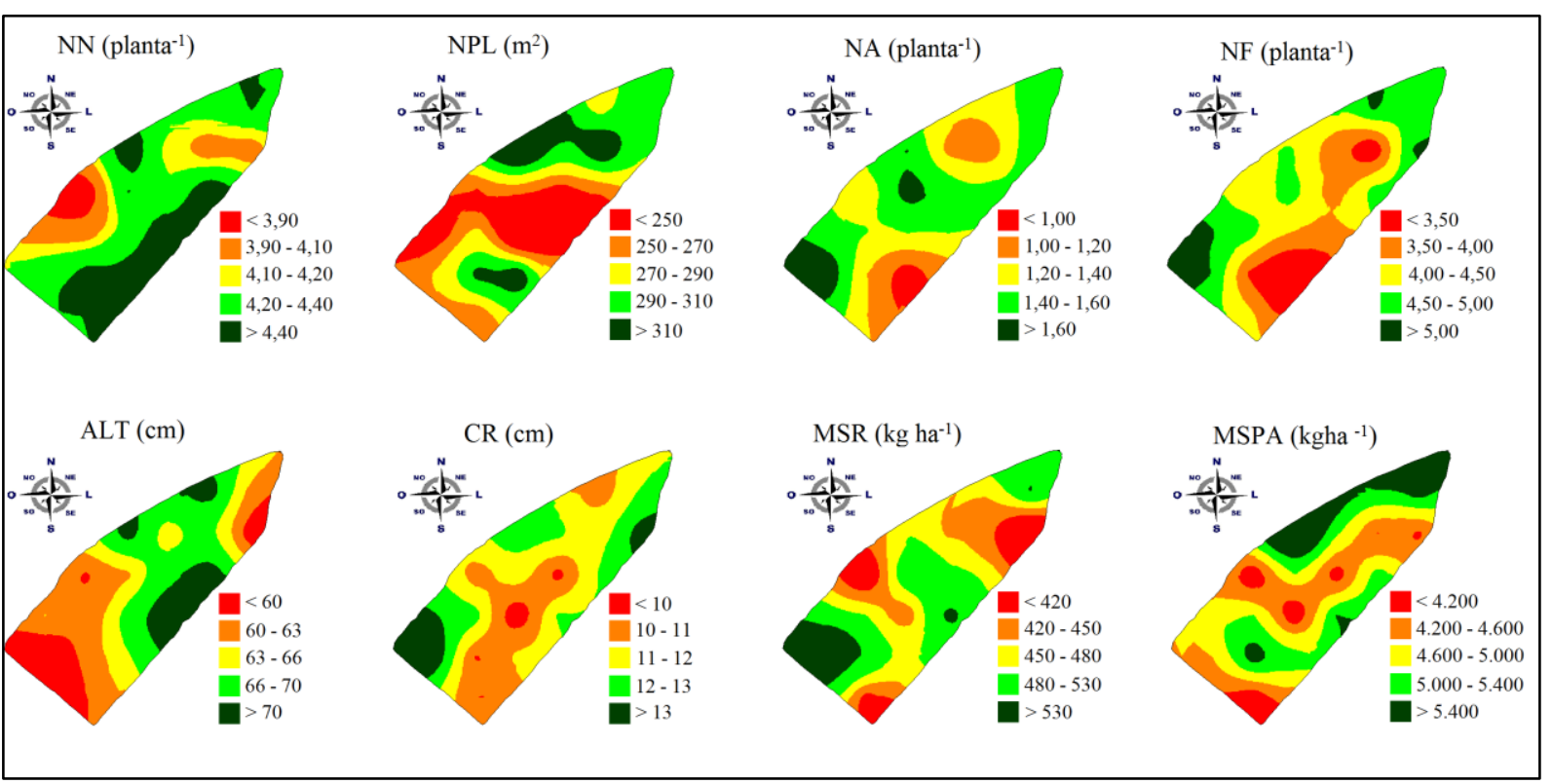

Figura 4 - Mapas temáticos do número de nós (NN), número de plantas (NPL), número de afilhos (NA), número de folhas por planta (NF), altura (ALT), comprimento radicular (CR), massa seca radicular (MSR) e massa seca de parte aérea (MSPA) da cultura do trigo aos 80 dias após a emergência. Palmeira das Missões, RS. 2016.

Figure 4 - Thematic maps of the number of nodes (NN), number of plants (NPL), number of tillers (NA), number of leaves per plant (NF), height (ALT), root length (CR), root dry weight (MSR) and dry mass of aerial part (MSPA) of wheat crop at 80 days after emergence. Palmeira das Missões, RS. 2016.

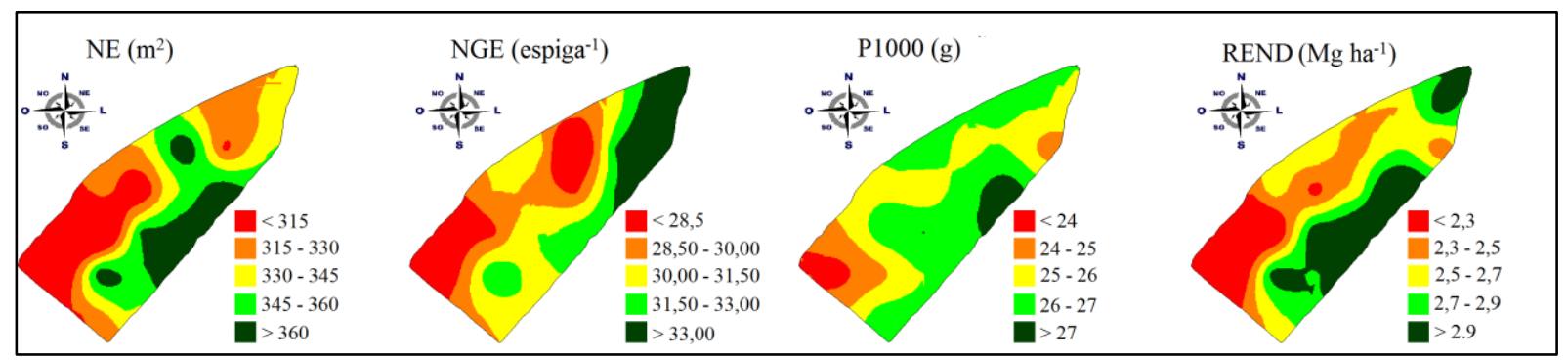

Figura 5 - Mapas temáticos do número de espigas (NE), número de grãos por espiga (NGE), peso de 1.000 grãos (P1000) e rendimento de grãos (REND) da cultura do trigo aos 130 dias após a emergência. Palmeira das Missões, RS. 2016.

Figure 5 - Thematic maps of the number of spikes (NE), number of grains per spike (NGE), 1000-grain weight (P1000) and grain yield (PROD) of wheat crop. Palmeira das Missões, RS. 2016.

\section{CONCLUSÃO}

Diferenças biométricas em plantas de trigo a partir do estádio fenológico 8 afetam os componentes do rendimento da cultura.

Plantas de trigo com elevada estatura (altura e número de nós no estádio 10,3) e reduzido comprimento radicular (estádio 8) apresentaram altos rendimentos de grãos.

\section{REFERÊNCIAS}

AMADO TJ et al. 2007. Variabilidade espacial e temporal da produtividade de culturas sob sistema plantio direto. Pesquisa Agropecuária Brasileira 42:1101-1110.

BREDEMEIER $\mathrm{C}$ et al. 2001. Efeito do tamanho das sementes de trigo no desenvolvimento inicial das plantas e no rendimento de grãos. Pesquisa Agropecuária Brasileira 36:1061-1068.

BRUM AL \& MÜLLER PK. 2008. A realidade da cadeia do trigo no Brasil: o elo produtores/cooperativas. Revista de Economia e Sociologia Rural 46:145-169. 
COELHO EC et al. 2009. Influência da densidade amostral e do tipo de interpolador na elaboração de mapas temáticos. Acta Science Agronomy 31:165-174.

COMISSÃO BRASILEIRA DE PESQUISA DE TRIGO E TRITICALE. 2011. Informações técnicas para trigo e triticale - safra 2012. Dourados: CPAO. 204p.

COMISSÃO DE QUÍMICA E FERTILIDADE DE SOLO (CQFS-RS/SC). 2004. Manual de adubação e calagem para os Estados do Rio Grande do Sul e Santa Catarina. 10.ed. Porto Alegre: Sociedade Brasileira de Ciência do Solo.

CONAB - COMPANHIA NACIONAL DE
ABASTECIMENTO. 2016. Séries históricas de safras-2016. Brasil. Disponível em: http://www.conab. gov.br/OlalaCMS/uploads/arquivos/16_04_07_10_39_11_ boletim_graos_abril_2016.pdf. Acesso em: 21 Mar. 2016. DALCHIAVON FC \& CARVALHO MP. 2012. Correlação linear e espacial dos componentes de produção e produtividade da soja. Semina: Ciências Agrárias 33:541552.

DAMIAN JM et al. 2016. Delineating management zones for precision agriculture applications: a case study on wheat in sub-tropical Brazil. Italian Agronomy Journal 11:171-179.

GAYLER S et al. 2014. Incorporating dynamic root growth enhances the performance of Noah-MP at two contrasting winter wheat field sites. Water Resources Research 50:1337-1356.

GEMTOS T et al. 2013. Precision Agriculture Application in Fruit Crops: Experience in Handpicked Fruits. Procedia Technology 8:324-332.

GOMES FP \& GARCIA CH. 2002. Estatística aplicada a experimentos agronômicos e florestais. Piracicaba: FEALQ. 309p.

GONDIM TCO et al. 2008. Análise de trilha para componentes do rendimento e caracteres agronômicos de trigo sob desfolha. Pesquisa Agropecuária Brasileira 43:487-493.

KHAMSS NN \& NAJAPHY A. 2012. Agro-morphological and phenological attributes under irrigated and rain-fed conditions in bread wheat genotypes. African Journal of Agricultural Research 7:51-57.

LARGE EC. 1954. Growth stages in cereals. Plant Pathology 3:128-129.

LONGNECKER $\mathrm{N}$ et al. 1993. Leaf emergence, tiller growth, and apical development of nitrogen-deficient spring wheat. Crop Science 33:154-160.

MAHAJAN GR et al. 2014. Using hyperspectral remote sensing techeniques to monitor nitrogen, phosphorus, sulphur and potassium in wheat (Triticum aestivum L.). Precision Agriculture 15:30-55.

MALUF JRT. 2000. Nova classificação climática do Estado do Rio Grande do Sul. Revista Brasileira de Agrometeorologia 8:141-150.

PEREIRA FS et al. 2011. Qualidade física de um Latossolo Vermelho submetido a sistemas de manejo avaliado pelos índices. Revista Brasileira de Ciência do Solo 35:87-95. RODRIGUEZ-MORENO $\mathrm{F}$ et al. 2014. Spatial interpretation of plant parameters in winter wheat. Precision Agriculture 15:447-465.

SANTOS HG et al. 2013. Sistema brasileiro de classificação de solos. Brasília: Embrapa. 353p.

SAS INSTITUTE - Statistical Analysis System. 1991. SAS/STAT User's Guide 8.0. North Caroline, NC: SAS Institute Inc.

WEBSTER R \& OLIVER MA. 2007. Geoestatitic for Enviromental Scientists. 2.ed. Chichester: John Wiley \& Sons Ltd.

USDA (UNITED STATES DEPARTMENT OF AGRICULTURE). 2014. Foreign Agricultural Service. Grain: World Markets and Trade. USA. Disponível em: <http://www.usda.gov/wps/portal/usda/usdahome $>$. Acesso em: 29 Mar. 2016.

VESOHOSKI $\mathrm{F}$ et al. 2011. Componentes do rendimento de grãos em trigo e seus efeitos diretos e indiretos na produtividade. Revista Ceres 58:337-341.

VIEIRA EA et al. 2007. Análise de trilha entre os componentes primários e secundários do rendimento de grãos em trigo. Revista Brasileira de Agrociência 13:169174.

ZHAO G et al. 2013. Impact of agricultural management practices on soil organic carbon: simulation of Australian wheat system. Global Change Biology 19:1585-1597.

ZUCOLOTO M et al. 2011. Correlação e variabilidade espacial de atributos químicos do solo e produção de bananeira 'prata-anã. Revista Brasileira de Fruticultura 33:479-484. 